Supporting Information:

\title{
Manipulation of Valley pseudospin Polarization by Selective Spin Injection in Chiral Two-Dimensional Perovskite/Monolayer Transition Metal Dichalcogenide Heterostructures
}

\author{
Yingying Chen ${ }^{1}$, Jiaqi Ma ${ }^{1}$, Zeyi Liu ${ }^{1}$, Junze $\mathrm{Li}^{1}$, Xiangfeng Duan ${ }^{2 *}$ and Dehui $\mathrm{Li}^{1 *}$ \\ ${ }^{1}$ School of Optical and Electronic Information and Wuhan National Laboratory for Optoelectronics, \\ Huazhong University of Science and Technology, Wuhan, 430074, China \\ ${ }^{2}$ Department of Chemistry and Biochemistry, University of California, Los Angeles, CA 90095, USA \\ *Correspondence to: Email: xduan@,chem.ucla.edu; dehuili@,hust.edu.cn.
}



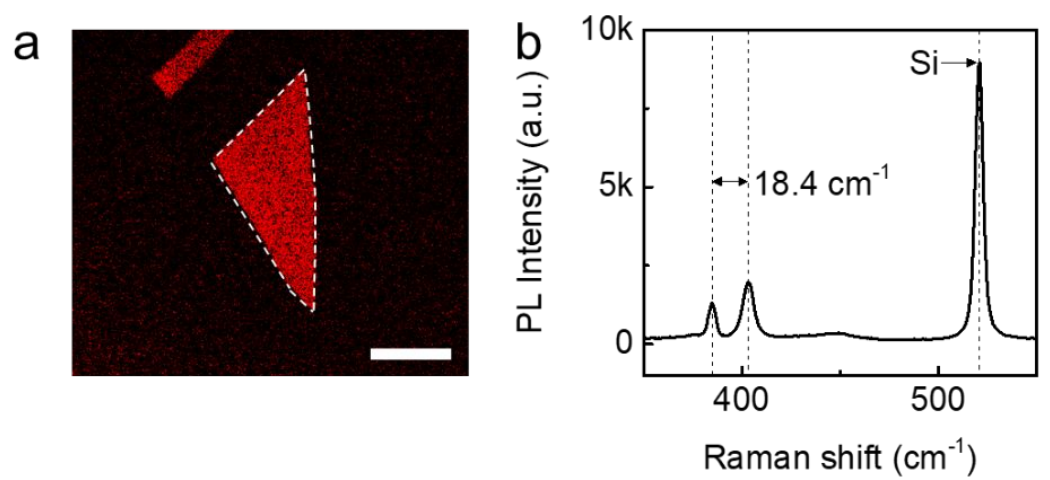

Figure S1. Fluorescence image (a) and Raman spectrum (b) of a monolayer $\mathrm{MoS}_{2}$ in Figure 1b. The excitation source is a $532 \mathrm{~nm}$ laser with the power of $178 \mu \mathrm{W}$. The scale bar is $10 \mu \mathrm{m}$. 

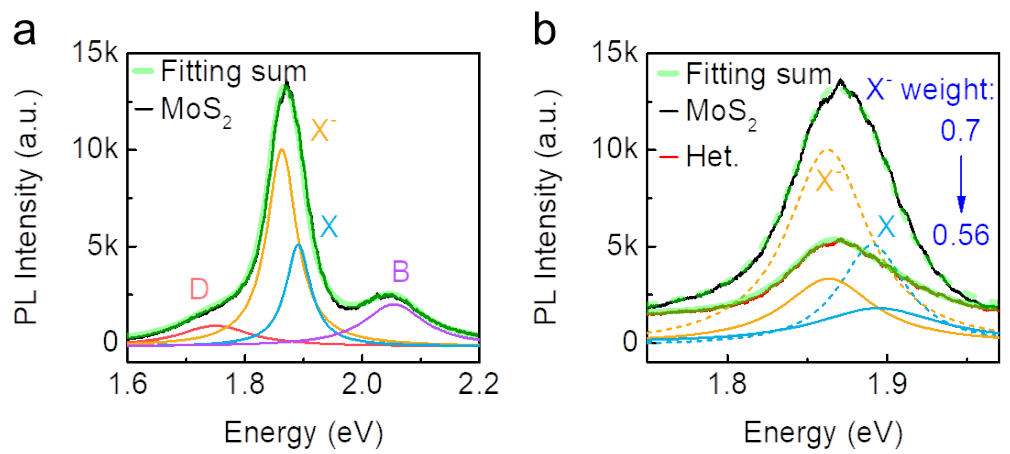

Figure S2. Lorentz fittings of PL spectra. (a) PL spectrum of a monolayer $\mathrm{MoS}_{2}$ fitted with four Lorentz peaks, which are neutral A exciton $(\mathrm{X})$, negatively charged $\mathrm{A}$ trion $\left(\mathrm{X}^{-}\right), \mathrm{B}$ exciton and defect-induced emission (D). (b) PL spectra of monolayer $\mathrm{MoS}_{2}$ and $\mathrm{MoS}_{2}$ heterostructure corresponding to Figure 1c. To be noticed, all spectra are fitted with four Lorentz peaks but only show the $\mathrm{X}$ and $\mathrm{X}^{-}$peaks. The $\mathrm{X}^{-}$weight can be determined by $\gamma=\frac{I_{X^{-}}}{I_{X^{-}}+I_{X}}$, where $\gamma$ is the $\mathrm{X}^{-}$ weight, $I_{X^{-}}$is the area of $\mathrm{X}^{-}$peak and $I_{X}$ is the area of $\mathrm{X}$ peak. 


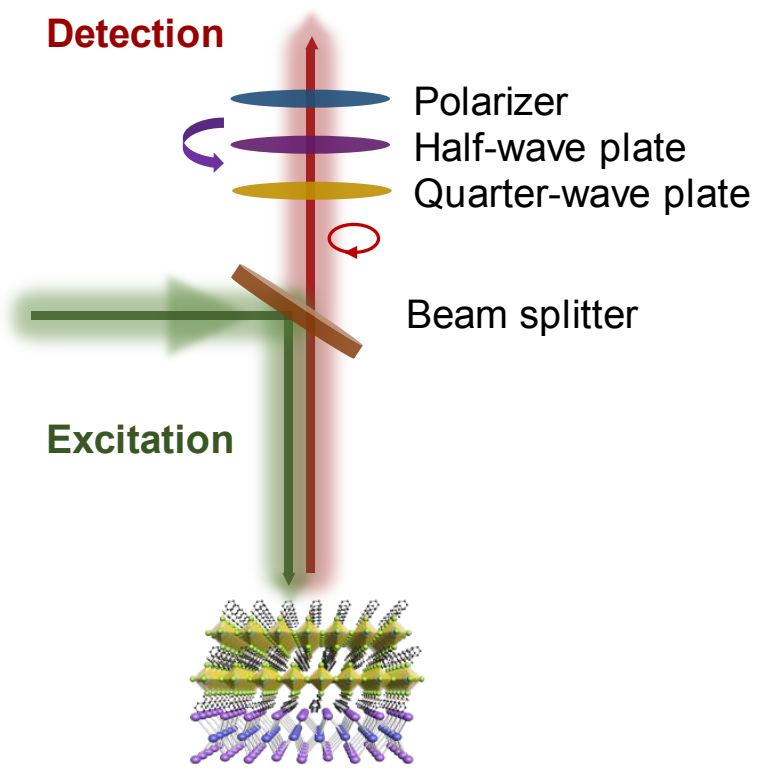

Figure S3. Optical setup of the polarization-resolved PL measurement. The excitation laser first passes through a beam splitter to excite the sample and then the emission signal was detected by a set of quarter-wave plate, half-wave plate and another polarizer. 

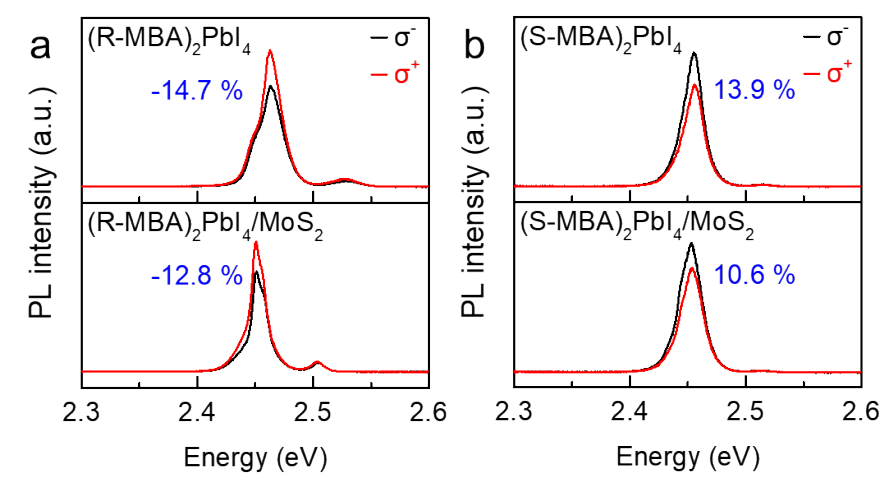

Figure S4. Polarization-resolved PL spectra of bare (R-MBA) $)_{2} \mathrm{PbI}_{4}$ and $(\mathrm{R}-\mathrm{MBA})_{2} \mathrm{PbI}_{4}$ in $(\mathrm{R}-$ $\mathrm{MBA})_{2} \mathrm{PbI}_{4} / \mathrm{MoS}_{2}(\mathrm{a})$, bare (S-MBA) $)_{2} \mathrm{PbI}_{4}$ and $(\mathrm{S}-\mathrm{MBA})_{2} \mathrm{PbI}_{4}$ in $(\mathrm{S}-\mathrm{MBA})_{2} \mathrm{PbI}_{4} / \mathrm{MoS}_{2}$ (b) excited by a linearly polarized $473 \mathrm{~nm}$ laser at $78 \mathrm{~K}$. The spectra are taken from other two representative devices different from the ones used in Figure 1 in the main text. 


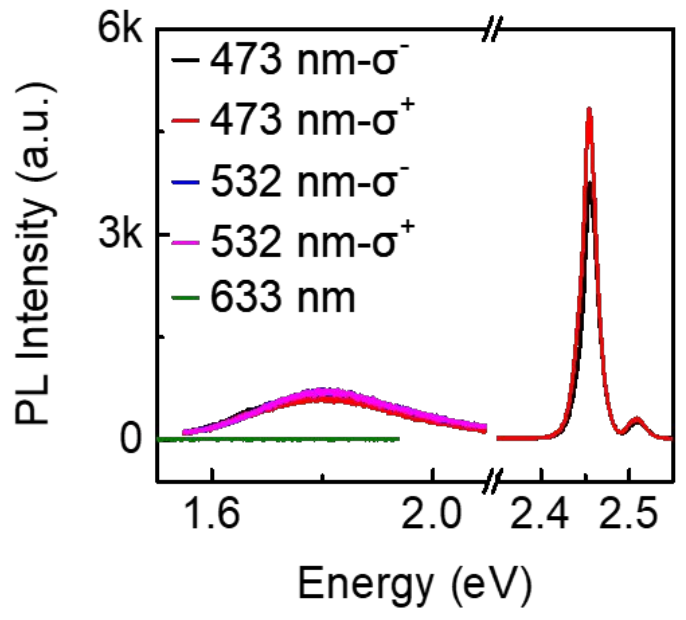

Figure S5. Polarization-resolved PL spectra of $(\mathrm{R}-\mathrm{MBA})_{2} \mathrm{PbI}_{4}$ excited by linearly polarized $473 \mathrm{~nm}, 532 \mathrm{~nm}$ and $633 \mathrm{~nm}$ lasers at $78 \mathrm{~K}$. 

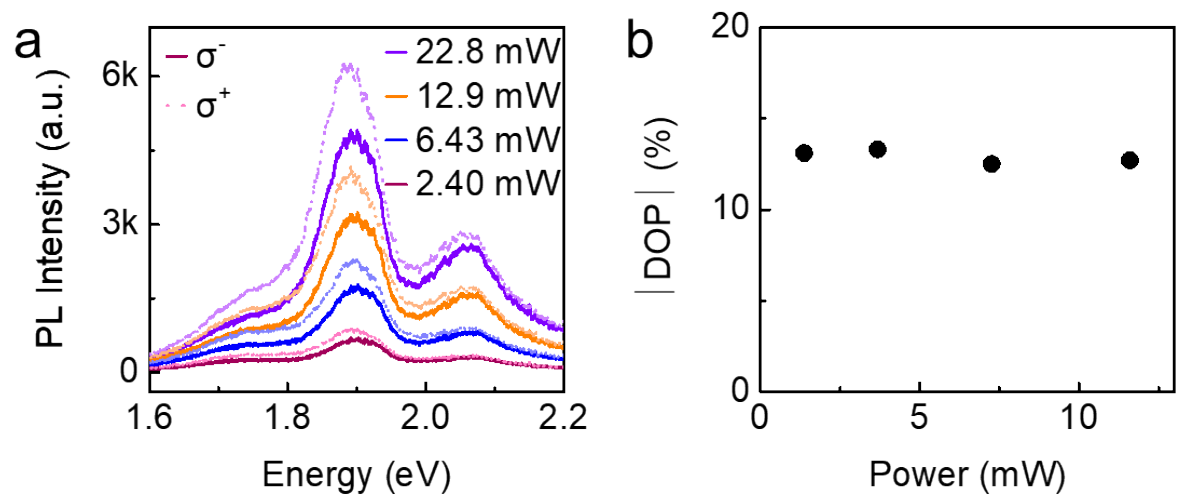

Figure S6. Power-dependent polarization-resolved PL (a) and the calculated absolute DOP (b) of a (S-MBA) $)_{2} \mathrm{PbI}_{4} / \mathrm{MoS}_{2}$ heterostructure at $78 \mathrm{~K}$ excited by a linearly polarized $532 \mathrm{~nm}$ laser with the power of $6.43 \mathrm{~mW}$. 

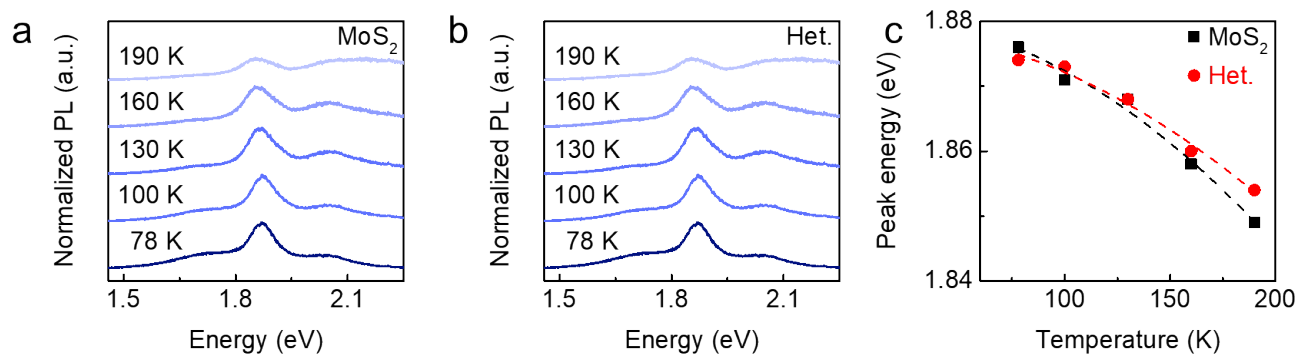

Figure S7. Temperature-dependent PL studies of a $(\mathrm{R}-\mathrm{MBA})_{2} \mathrm{PbI}_{4} / \mathrm{MoS}_{2}$ heterostructure.

Temperature-dependent spectra of monolayer $\mathrm{MoS}_{2}$ (a) and the (R-MBA) ${ }_{2} \mathrm{PbI}_{4} / \mathrm{MoS}_{2}$ heterostructure (Het.) (b) excited by a linearly polarized $532 \mathrm{~nm}$ laser with the power of $6.43 \mathrm{~mW}$. (c) The peak position shifts of A exciton in $\mathrm{MoS}_{2}$ and the (R-MBA) $)_{2} \mathrm{PbI}_{4} / \mathrm{MoS}_{2}$ heterostructure versus temperature. The black and red dashed lines are fitted by the Varshni equation, ${ }^{1,2}$ which describes the band gap evolution with temperature. 

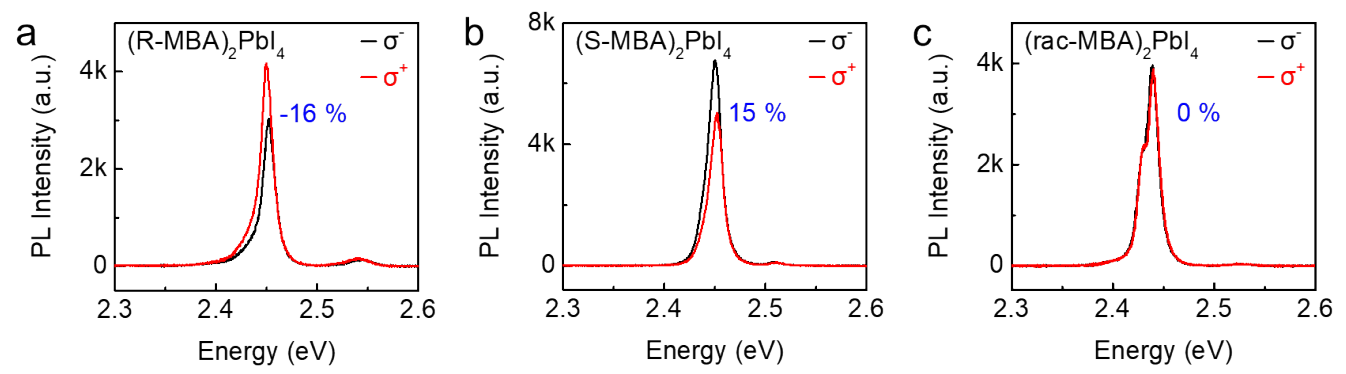

Figure S8. Polarization-resolved PL spectra of $\left(\mathrm{R}-\mathrm{MBA}_{2} \mathrm{PbI}_{4}(\mathrm{a}),(\mathrm{S}-\mathrm{MBA})_{2} \mathrm{PbI}_{4}(\mathrm{~b})\right.$ and (racMBA) $)_{2} \mathrm{PbI}_{4}(\mathrm{c})$ microplates used in Figure 4a excited by a linearly polarized $473 \mathrm{~nm}$ laser with the power of $0.02 \mu \mathrm{W}$ at $78 \mathrm{~K}$. 


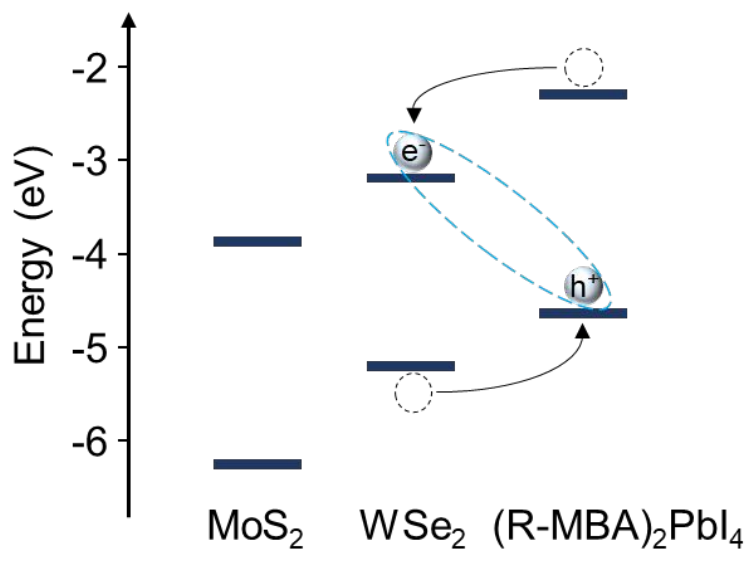

Figure S9. Band alignment of monolayer $\mathrm{MoS}_{2}$, monolayer $\mathrm{WSe}_{2}$ and chiral 2D perovskite as well as the charge transfer and recombination process in the $(\mathrm{R}-\mathrm{MBA})_{2} \mathrm{PbI}_{4} / \mathrm{WSe}_{2}$ heterostructure. 

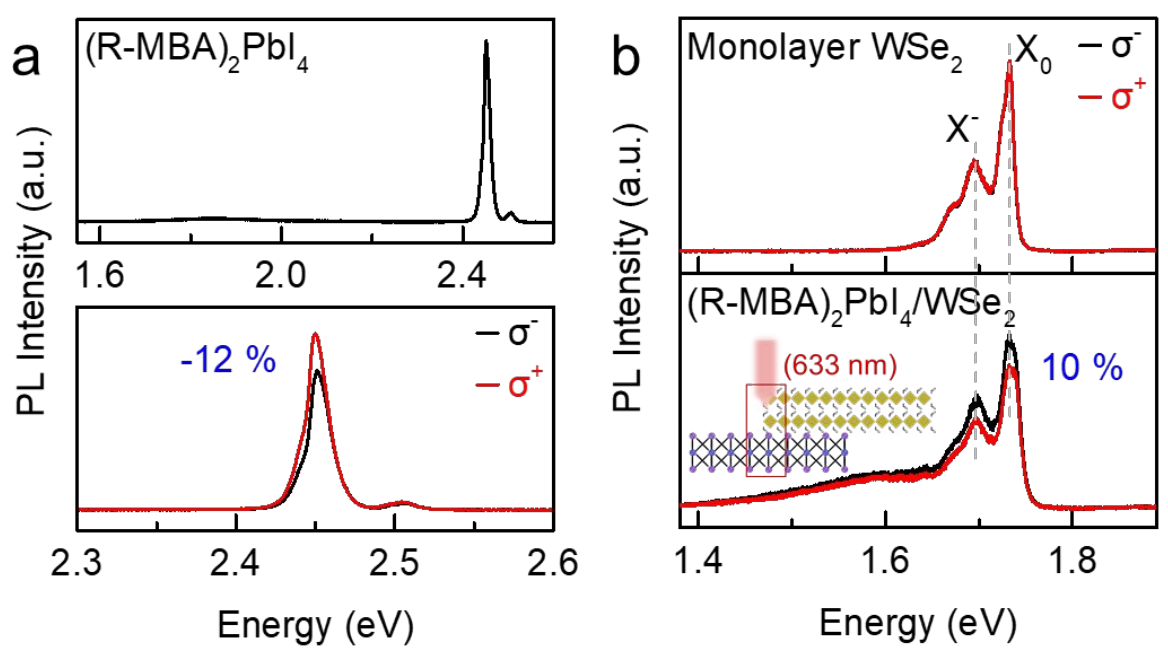

Figure S10. PL spectra of an edge excited (R-MBA) $)_{2} \mathrm{PbI}_{4} / \mathrm{WSe}_{2}$ heterostructure at $78 \mathrm{~K}$. (a) PL spectrum of (R-MBA) $)_{2} \mathrm{PbI}_{4}$ in the upper panel and its CPL emission spectra in the lower panel excited by a linearly polarized $473 \mathrm{~nm}$ laser with the power of $0.02 \mu \mathrm{W}$. (b) Polarization-resolved $\mathrm{PL}$ of the constituent monolayer $\mathrm{WSe}_{2}$ (upper panel) and the (R-MBA) $)_{2} \mathrm{PbI} / \mathrm{WSe}_{2}$ heterostructure (lower panel) excited by a linearly polarized $633 \mathrm{~nm}$ laser with the power of $485 \mu \mathrm{W}$. Inset: schematic illustration of the edge excitation with the excitation region highlighted by the red box. 


\section{References}

1. Varshni, Y. P. Temperature Dependence of the Energy Gap in Semiconductors. Physica 1967, 34, 149-154.

2. Korn, T.; Heydrich, S.; Hirmer, M.; Schmutzler, J.; Schüller, C. Low-Temperature Photocarrier Dynamics in Monolayer $\mathrm{MoS}_{2}$. Appl. Phys. Lett. 2011, 99, 102109. 